\title{
Qualitatively Stable Nonstandard Finite Difference Scheme for Numerical Solution of the Nonlinear Black-Scholes Equation
}

\author{
Mohammad Mehdizadeh Khalsaraei $\mathbb{D D}^{1}{ }^{1}$ Ali Shokri $\mathbb{D}^{1},{ }^{1}$ Zahra Mohammadnia ${ }^{\mathbb{D}},{ }^{1}$ \\ and Hamid Mohammad Sedighi $\mathbb{D}^{2}$ \\ ${ }^{1}$ Department of Mathematics, Faculty of Science, University of Maragheh, Maragheh, Iran \\ ${ }^{2}$ Mechanical Engineering Department, Faculty of Engineering, Shahid Chamran University of Ahvaz, Ahvaz, \\ P.O. Box 61357-43337, Iran
}

Correspondence should be addressed to Mohammad Mehdizadeh Khalsaraei; mehdizadeh@maragheh.ac.ir

Received 19 December 2020; Accepted 26 April 2021; Published 11 May 2021

Academic Editor: Efthymios G. Tsionas

Copyright (C) 2021 Mohammad Mehdizadeh Khalsaraei et al. This is an open access article distributed under the Creative Commons Attribution License, which permits unrestricted use, distribution, and reproduction in any medium, provided the original work is properly cited.

In this paper, we use a numerical method for solving the nonlinear Black-Scholes partial differential equation of the European option under transaction costs, which is based on the nonstandard discretization of the spatial derivatives. The proposed scheme, in addition to the unconditional positivity, is stable, consistent, and monotone. In order to illustrate the efficiency of the new method, numerical results have been performed by four models.

\section{Introduction}

Financial mathematics is a branch of applied mathematics that deals with financial markets. Option contracts are the most traditionally financial instruments. Hence, with the increasing demand for this financial instrument, the pricing of contracts has very important role to play in the economy. An option on an underlying asset traded on financial markets is an agreement that gives its holder the option to buy or sell the asset mentioned in the contract on a specified date at a certain and predetermined price. The date specified in the contract also calls to the maturity date or expiration date, and the price specified in the contract is called the strike price or exercise price. The biggest advantage of an option contract is limited loss and unlimited profits. Option contracts are divided into two types such as European and American types according to the time of exercise. A European option is only applicable at the time specified in the contract, but the American option is applicable at any time until maturity date $t \leq T$. One of the models in the discussion of financial derivatives pricing is the Black-Scholes model. This model was introduced by Fisher Black and Miran
Scholes and developed by Robert Merton [1]. It is represented by a partial differential equation in the following form:

$$
U_{t}+\frac{1}{2} \sigma_{0}^{2} S^{2} U_{S S}+r S U_{S}-r U=0,
$$

where $U=U(S, t)$ is the value of the option, $S$ is the current underlying asset price, $t \mathrm{t}=\mathrm{t}$ is the time, $T>0$ is the expiration date of the option, $r \geq 0$ is the risk-free interest rate, and $\sigma_{0}$ is the volatility (also called implicit volatility). $r$ and $\sigma_{0}$ are fixed. Partial differential equation paid attention in the field of economics with the Black-Scholes model. Therefore, in order to obtain the price of these contracts, we need a simple and accurate method to solve this differential equation. The standard Black-Scholes equation is considered in a complete market under certain simple assumptions such as the cash market, without transaction cost. Also, the most important feature in the classical Black-Scholes model, the volatility of an underlying asset is assumed to be constant. In 1987, all over the world, stock the value decreased observably in the short course of time. After this decrease, analysts have concluded that the options with profits and 
losses are traded with higher implicit volatility versus the option that do not have profit and loss. So, this fact is not compatible with the assumption of constant volatility in the Black-Scholes model. In addition to despite the transaction cost, the Black-Scholes equation changes to an equation with a nonlinear volatility function, which depends on the time, current underlying asset price, and the price derivatives of the option relative to the underlying asset price. Then, the classic Black-Scholes equation changes to the following nonlinear Black-Scholes equation:

$$
U_{t}+\frac{1}{2} \sigma^{2}\left(t, S, U_{S}, U_{S S}\right) S^{2} U_{S S}+r S U_{S}-r U=0
$$

in which $\sigma$ is the modified volatility. Various nonlinear volatility models have been presented in [2-4]. We consider the nonlinear Barles and Soner model which is obtained as follows:

$$
\sigma^{2}=\sigma_{0}^{2}\left(1+\Psi\left[e^{r(T-t)} a^{2} S^{2} U_{S S}\right]\right),
$$

where $a=\mu \sqrt{\gamma N}$, in which $\gamma$ is the risk-adjusted factor and $N$ is the number of options that are sold. The function $\Psi$ is the solution of the following nonlinear initial value problem [5]:

$$
\Psi^{\prime}(w)=\frac{\Psi(w)+1}{2 \sqrt{w \Psi(w)}-w}, \quad w \neq 0, \Psi(0)=0
$$

For function $\Psi$, an exact implicit solution is presented as follows [6]:

$$
\begin{gathered}
\sqrt{w}=\frac{-\sinh ^{-1} \sqrt{\Psi}}{\sqrt{\Psi+1}}+\sqrt{\Psi}, \quad \text { for } w>0, \Psi(w)>0, \\
\sqrt{-w}=\frac{\sin ^{-1} \sqrt{-\Psi}}{\sqrt{\Psi+1}}-\sqrt{-\Psi}, \quad \text { for } w<0,-1<\Psi(w)<0 .
\end{gathered}
$$

Let us consider equation (2) with the nonlinear modified volatility of Barles and Soner (3) and (4). By applying the variable $\tau=T-t$, equation (1) is converted to the following equation:

$$
V_{\tau}=\frac{1}{2} \sigma^{2}\left(V_{S S}\right) S^{2} V_{S S}+r S V_{S}-r V
$$

for $S>0$ and $0<\tau \leq T$,

$$
\sigma^{2}\left(V_{S S}\right)=\sigma_{0}^{2}\left(1+\Psi\left[e^{r \tau} a^{2} S^{2} V_{S S}\right]\right)
$$

as in [5] we consider the initial condition, the first boundary condition, and the second boundary condition for $S \in\left(0, S_{\max }\right)$ and $\tau \in(0, T]$ as follows:

$$
\begin{aligned}
& L_{1}(S)= \begin{cases}\max (S-K, 0), & \text { for the Call option, } \\
\max (K-S, 0), & \text { for the Put option, } \\
\max \left(S-K_{1}, 0\right)-2 \max \left(S-K_{2}, 0\right)+\max \left(S-K_{3}, 0\right), & \text { for Butterfly Spread, } \\
\text { for Cash or Nothing, }\end{cases} \\
& L_{2}(\tau)= \begin{cases}0, & \text { for the Call option, } \\
K e^{-r \tau}, & \text { for the Put option, } \\
0, & \text { for Butterfly Spread, } \\
0, & \text { for Cash or Nothing, }\end{cases} \\
& L_{3}(\tau)= \begin{cases}S_{\max }-K e^{-r \tau}, & \text { for the Call option, } \\
0, & \text { for the Put option, } \\
0, & \text { for Butterfly Spread, } \\
B e^{-r \tau}, & \text { for Cash or Nothing, }\end{cases}
\end{aligned}
$$

where $K, K_{1}, K_{2}$, and $K_{3}$ denote the strike prices of the options, $B$ is a positive constant, and $H$ is the Heaviside function. Conditions $L_{1}(0)=L_{2}(0)$ and $L_{1}\left(S_{\max }\right)=L_{3}(0)$ are established.

So far, no classical solution has been given for equation (2), but the viscosity solution is used as the solution of equation (2).

The numerical solution of the nonlinear Black-Scholes equation has been considered in some works. For example, in [7], an explicit method has been presented that preserves positivity property and is unconditional stable but not consistent under any conditions. A variational principle is established for the generalized $\mathrm{KdV}$-Burgers equation by the semiinverse method in [8-11]. In [12-14], the homotopy perturbation method is designed to obtain a solution to the Black-Scholes equation with boundary conditions for a European option pricing problem and nonlinear problems. Methods based on the variational iteration method (VIM) in [15-17] are proposed for the Black-Scholes model. In [18], an explicit nonstandard finite difference method has been 
proposed that it is stable and conditionally acceptable. Also, a finite difference method has been proposed which is the stability condition for it and is established by limiting the length of the step time and the spatial step size. In [5], a completely implicit time step method has been presented for (6) and represented convergence approximate solution to the viscosity solution and used Newton's algorithm to solve a nonlinear system. They caused numerical instability in the solution that has been resolved by an iterative method. This process leads to very expensive computing costs. Also in [6], the MOL method has been used to solve $(6)$. In $[19,20]$, equation (6) is solved by the variable $x=\ln (S / K)$ which in addition to having very expensive computational cost, convergence, and other results of this work is under relatively unrealistic conditions or limits the step-size time and space.

In [21], considering a class of nonlinear option pricing models, Newton and Picard iterative procedures are proposed for solving the nonlinear systems of algebraic equations. Also, to improve the computational fast, two-grid algorithms are developed. In [22], combination of a sixthorder finite difference scheme in space and a third-order strong stability preserving Runge-Kutta in time has been used to obtain numerical solutions of the linear and nonlinear European put option models. In [23], a new fourthorder finite difference approximation is contributed and equipped with the fourth-order Runge-Kutta scheme for the nonlinear Black-Scholes. They proved that under several criteria, the procedure is time stable. Also, the proposed technique reduces the computational cost when more accurate results are requested. In [24], nonlinear generalization of the Black- Scholes equation for pricing and American-style call options is investigated. They proposed a numerical method that involves transforming the free boundary problem for a nonlinear Black-Scholes equation into the so-called Gamma variational inequality with a new variable depending on the Gamma of the option. Also, a modified projected successive overrelaxation method in order to construct a numerical scheme for discretization of the Gamma variational inequality is presented.

In this paper, we solve equation (6) by using an explicit method that has very low computational cost, and the method is free of any spurious oscillations. It is unconditionally positive, stable, consistent, and monotone. We show that the solution of the proposed method converges to the solution of the viscosity of the equation.

The rest of the paper is organized as follows. In section 2, we give a brief summary of the NSFD methods. In section 3, we examine the application of nonstandard methods through spatial nonlocalized discretization for the nonlinear Black-Scholes under transaction costs. In section 4, we analyze the new method. The numerical results are given in section 5 .

\section{Nonstandard Finite Difference Scheme}

In this section, we give some preliminaries including nonstandard finite difference methods. The initial foundation of NSFD schemes came from the exact finite difference schemes. Numerical methods based on the standard finite difference approach are consistent with the original differential equation and guarantee convergence of the discrete solution to the exact one, but they impose severe restrictions on the time step, and essential qualitative properties of the solution are not transferred to the numerical solution. One response to this situation was the initiation by Mickens [25] of a research program for the investigation of new methods for constructing finite difference schemes that are convergent for any step size. Nonstandard finite difference methods (NSFDs) in addition to the usual properties of consistency, stability, and hence convergence produce numerical solutions that also exhibit essential properties of solutions $[3-5,13,15,16,18,21,26-38]$.

This class of schemes and their formulations centers on two issues: first, how should discrete representations for derivatives be determined and second what are the proper forms to be used for nonlinear and reaction terms. The forward finite difference method is one of the simplest discretization schemes. In this method, the derivative $V_{x}$ is replaced by $(V(x+h)-V(x) / h)$, where $h$ is step size. However, in the Mickens schemes, this term is replaced by $(V(x+h)-V(x) / \phi(h))$, where $\phi(h)$ is an increasing continuous function of $h$, and the function $\phi(h)$ satisfies the following conditions:

$$
\phi(h)=h+O\left(h^{2}\right), \quad 0<\phi(h)<1, h \longrightarrow 0 .
$$

Note that in taking $\lim h \longrightarrow 0$ to obtain the derivative, the use of any of this $\phi(h)$ will lead to the usual result for the first derivative:

$$
\frac{\mathrm{d} V}{\mathrm{~d} x}=\lim _{h \longrightarrow 0} \frac{V\left(x+\phi_{1}(h)\right)-V(x)}{\phi_{2}(h)}=\lim _{h \longrightarrow 0} \frac{V(x+h)-V(x)}{h},
$$

where $\phi_{1}(h)$ and $\phi_{2}(h)$ are continuous functions of the step size $h$. A scheme is called a nonstandard finite difference method if at least one of the following conditions is met:

(i) In the discrete derivatives, the traditional denominator $h$ is replaced by a nonnegative function $\phi(h)$ such that

$$
\phi(h)=h+O\left(h^{2}\right), \quad 0<\phi(h)<1, h \longrightarrow 0,
$$

or

$\phi\left(h^{2}\right)=h^{2}+O\left(h^{4}\right), \quad 0<\phi\left(h^{2}\right)<1, h \longrightarrow 0$.

Examples of functions $\phi(h)$ that satisfy these conditions are as follows [25]:

$$
h, 1-e^{-h}, \sin (h), \text { or } 4 \sin ^{2}\left(\frac{h}{2}\right) .
$$

(ii) Nonlinear terms are approximated in a nonlocal way, i.e., by a suitable function of several points of the mesh. If there are reaction terms of the differential equation $[3-5,13,15,16,18,21,26-28]$, these are replaced by 


$$
\begin{aligned}
V & \approx 3 V_{j+1}^{n}-2 V_{j}^{n}, \\
V & \approx \frac{2 V_{j+1}^{n}+V_{j}^{n}}{3}, \\
V^{2} & \approx a\left(V_{j}^{n}\right)^{2}+b V_{j}^{n} V_{j+1}^{n}, \quad a+b=1, a, b \in \mathbb{R} .
\end{aligned}
$$

One can say that there is no appropriate general method to choose the function $\phi(h)$ or to choose which nonlinear terms are to be replaced, and some special techniques may be found in $[3-5,13,15,16,18,21,26-28]$.

\section{Construction of the New NSFD}

In this section, by using the mentioned rules for NSFD schemes, we propose our new NSFD scheme. Let us consider the computational domain $\Omega=\left[0, S_{\max }\right] \times[0, T]$ and discretize it in the following form. We introduce a grid of mesh points $(S, t)=\left(S_{j}, t_{n}\right) \quad$ where $S_{j}=j h$ and $t_{n}=n \Delta t$, $j=0,1, \ldots, M ; n=0,1, \ldots, X$, and the spatial step-size is given by $h=S_{\max } / M$, and the time step size is $\Delta t=T / X$. We denote the approximation of $V\left(S_{j}, t_{n}\right)$ by $V_{j}^{n}$. The new NSFD is of the following form:

$$
-\frac{V_{j}^{n+1}-V_{j}^{n}}{\Delta \tau}+\frac{1}{2} \sigma^{2}\left(\left(V_{S S}^{n}\right)(j)\right) S_{j}^{2} \frac{V_{j-1}^{n}-2 V_{j}^{n+1}+V_{j+1}^{n}}{h^{2}}+r S_{j} \frac{V_{j+1}^{n}-V_{j}^{n+1}}{h}-r V_{j}^{n+1}=0 .
$$

The explicit form of (17) is as follows:

$$
V_{j}^{n+1}=\frac{(1 / 2)\left(\sigma^{2}\left(\left(V_{S S}^{n}\right)(j)\right) S_{j}^{2} / h^{2}\right) V_{j-1}^{n}+(1 / \Delta \tau) V_{j}^{n}+\left(\left(r S_{j} / h\right)+(1 / 2)\left(\sigma^{2}\left(\left(V_{S S}^{n}\right)(j)\right) S_{j}^{2} / h^{2}\right)\right) V_{j+1}^{n}}{(1 / \Delta \tau)+\left(r S_{j} / h\right)+\left(\sigma^{2}\left(\left(V_{S S}^{n}\right)(j)\right) S_{j}^{2} / h^{2}\right)+r} .
$$

The finite difference approximation provides a difference equation as follows:

$$
V^{n+1}=A^{n}\left(V^{n}\right) V^{n}+B^{n}
$$

with (8)-(10), and we define the initial and boundary conditions as follows:

$$
\begin{aligned}
V_{j}^{0} & =L_{1}\left(S_{j}\right), \\
V_{0}^{n} & =L_{2}\left(\tau_{n}\right), \\
V_{M}^{n} & =L_{3}\left(\tau_{n}\right) .
\end{aligned}
$$

The matrix form (18) for all $j=1, \ldots, M-1$ and $n=$ $0,1, \ldots, N-1$ is given in the following form:
$A^{n}\left(V^{n}\right)=\left(\begin{array}{ccccccc}y_{1}^{n} & z_{1}^{n} & 0 & \cdots & 0 & 0 & 0 \\ x_{2}^{n} & y_{2}^{n} & z_{2}^{n} & \cdots & 0 & 0 & 0 \\ 0 & x_{3}^{n} & y_{3}^{n} & \cdots & 0 & 0 & 0 \\ \vdots & \vdots & \vdots & \ddots & \vdots & \vdots & \vdots \\ 0 & 0 & 0 & \cdots & y_{M-3}^{n} & z_{M-3}^{n} & 0 \\ 0 & 0 & 0 & \cdots & x_{M-2}^{n} & y_{M-2}^{n} & z_{M-2}^{n} \\ 0 & 0 & 0 & \cdots & 0 & x_{M-1}^{n} & y_{M-1}^{n}\end{array}\right)$, $B^{n}=\left(\begin{array}{c}x_{1}^{n} V_{0}^{n} \\ 0 \\ 0 \\ \vdots \\ 0 \\ 0 \\ z_{M-1}^{n} V_{M}^{n}\end{array}\right)$ 
in which $A^{n}\left(V^{n}\right)$ is a tridiagonal matrix with positive components and $B^{n}$ is known as boundary values. The new scheme can be written in the following form:

$$
V_{j}^{n+1}=x_{j}^{n} V_{j-1}^{n}+y_{j}^{n} V_{j}^{n}+z_{j}^{n} V_{j+1}^{n} \text {, }
$$

where

$$
\begin{gathered}
x_{j}^{n}\left(V^{n}\right)=\frac{\left(\sigma^{2}\left(\left(V_{S S}^{n}\right)(j)\right) S_{j}^{2} / 2 h^{2}\right)}{(1 / \Delta \tau)+\left(r S_{j} / h\right)+\left(\sigma^{2}\left(\left(V_{S S}^{n}\right)(j)\right) S_{j}^{2} / h^{2}\right)+r}, \\
y_{j}^{n}\left(V^{n}\right)=\frac{(1 / \Delta \tau)}{(1 / \Delta \tau)+\left(r S_{j} / h\right)+\left(\sigma^{2}\left(\left(V_{S S}^{n}\right)(j)\right) S_{j}^{2} / h^{2}\right)+r}, \\
z_{j}^{n}\left(V^{n}\right)=\frac{\left(r S_{j} / h\right)+(1 / 2)\left(\sigma^{2}\left(\left(V_{S S}^{n}\right)(j)\right) S_{j}^{2} / h^{2}\right)}{(1 / \Delta \tau)+\left(r S_{j} / h\right)+\left(\sigma_{j}^{2}\left(\left(V_{S S}^{n}\right)(j)\right) S_{j}^{2} / h^{2}\right)+r} .
\end{gathered}
$$

\section{Analysis of the New Method}

In this section, we investigate the monotonicity, positivity preserving, stability, consistency, and convergency properties of the new method.
For $j=1, \ldots, M-1$ and $n=0,1, \ldots, N-1$, we define the function $\varphi_{j}^{n+1}$ as follows:

$$
\varphi_{j}^{n+1}\left(V_{j}^{n+1}, V_{j+1}^{n}, V_{j-1}^{n}, V_{j}^{n}\right):=p_{j} V_{j+1}^{n}+q_{j} V_{j}^{n+1}-\frac{1}{\Delta \tau_{n}} V_{j}^{n}-\frac{1}{2} S_{j}^{2} \sigma^{2}\left(\left(V_{S S}^{n}\right)(j)\right)\left(V_{S S}^{n}\right)(j)=0,
$$

where

$$
\begin{aligned}
& p_{j}=-\frac{r S_{j}}{h_{j}}, \\
& q_{j}=\frac{1}{\Delta \tau_{n}}+\frac{r S_{j}}{h_{j}}+r .
\end{aligned}
$$

Lemma 1 (monotonicity). The new NSFD method is monotone.

Proof. As in [5] which is enough to show that for each $\xi>0$ and $j=1, \ldots, M-1$,

$$
\begin{aligned}
& \varphi_{j}^{n+1}\left(V_{j}^{n+1}, V_{j+1}^{n}+\xi, V_{j-1}^{n}+\xi, V_{j}^{n}+\xi\right) \leq \varphi_{j}^{n+1}\left(V_{j}^{n+1}, V_{j+1}^{n}, V_{j-1}^{n}, V_{j}^{n}\right), \\
& \varphi_{j}^{n+1}\left(V_{j}^{n+1}+\xi, V_{j+1}^{n}, V_{j-1}^{n}, V_{j}^{n}\right) \geq \varphi_{j}^{n+1}\left(V_{j}^{n+1}, V_{j+1}^{n}, V_{j-1}^{n}, V_{j}^{n}\right) .
\end{aligned}
$$

Since $p_{j} \leq 0,\left(1 / \Delta \tau_{n}\right)>0$, and $q_{j}>0$, the first three sentences of the right of the relationship (26), respectively, are nonincreasing in $V_{j+1}^{n+1}$, increasing in $V_{j}^{n+1}$, and decreasing in $V_{j}^{n}$.

Suppose $E_{k}=(0,0, \ldots, \underbrace{1}_{k \text { h }}, 0, \ldots, 0)^{T}$ is a column vector $(M-1) \times 1$. We have $k$ th

$$
\begin{aligned}
\left(V_{S S}\left(V^{n}+\xi E_{j-1}+\xi E_{j+1}\right)\right)(j) & =\frac{1}{h^{2}}\left(V_{j-1}^{n}+\xi\right)-\frac{2}{h^{2}} V_{j}^{n+1}+\frac{1}{h^{2}}\left(V_{j+1}^{n}+\xi\right) \\
& =\left(V_{S S}^{n}\right)(j)+\frac{2}{h^{2}} \xi \\
\left(V_{S S}\left(V^{n}+\xi E_{j}\right)\right)(j) & =\frac{1}{h^{2}} V_{j-1}^{n}-\frac{2}{h^{2}}\left(V_{j}^{n+1}+\xi\right)+\frac{1}{h^{2}} V_{j+1}^{n}=\left(V_{S S}^{n}\right)(j)-\frac{2}{h^{2}} \xi .
\end{aligned}
$$

Assume that we take into account the nonlinear sentence is to the right of the relation (26). From (3), we have

$$
\frac{1}{2} S_{j}^{2} \sigma^{2}\left(v_{j}^{n}\right) v_{j}^{n}=\frac{1}{2} \sigma_{0}^{2} S_{j}^{2}\left[1+\Psi\left(K_{j}^{n+1} v_{j}^{n}\right)\right] v_{j}^{n}
$$


where

$$
v_{j}^{n}=\left(V_{S S}\right)(j), \quad K_{j}^{n+1}=e^{r \tau_{n+1}} a^{2} S_{j}^{2}>0,
$$

for each $j=0,1, \ldots, M-1$ and $K>0$; independent of $v$, by derivative phrase $(1+\Psi(K v))$ relative to the variable $v$ and using (5) and (24) and $w=K v$, we have

$$
\begin{aligned}
\frac{\mathrm{d}}{\mathrm{d} v}[(1+\Psi(K v)) v] & =\Psi^{\prime}(K v)(K v)+(1+\Psi(K v)) \\
& =\frac{1+\Psi(w)}{2 \sqrt{w \Psi(w)}-w} w+(1+\Psi(w)) \\
& =(1+\Psi(w)) \frac{2 \sqrt{w \Psi(w)}}{2 \sqrt{w \Psi(w)}-w} \\
& =2 \sqrt{w \Psi(w)} \Psi^{\prime}(w) \geq 0
\end{aligned}
$$

So, $(1+\Psi(K v)) v$ is an increasing function of $v$. Using the above symbols and by monotone combination of $(1+$ $\Psi(K v)) v$ and properties of the linear sentences of the relationship (26) and (30), we have

$$
\begin{aligned}
\varphi_{j}^{n+1}\left(V_{j}^{n+1}, V_{j+1}^{n}+\xi, V_{j-1}^{n}+\xi, V_{j}^{n}+\xi\right)= & p_{j}\left(V_{j+1}^{n}+\xi\right)+q_{j} V_{j}^{n+1} \\
& -\frac{1}{\Delta \tau_{n}}\left(V_{j}^{n}+\xi\right)-\frac{1}{2} \sigma_{0}^{2} S_{j}^{2}\left[1+\Psi\left(K_{j}^{n+1}\left(v_{j}^{n}+\frac{2}{h^{2}} \xi\right)\right)\right]\left(v_{j}^{n}+\frac{2}{h^{2}} \xi\right) \\
\leq & \varphi_{j}^{n+1}\left(V_{j}^{n+1}, V_{j+1}^{n}, V_{j-1}^{n}, V_{j}^{n}\right) .
\end{aligned}
$$

This is the same as (28). Similarly from monotonicity $(1+\Psi(K v)) v$ and (31), it can be shown that (29) is confirmed, and this completes the proof.

Now, we investigate the positivity property of the constructed methods in the previous section. With positivity, we mean that the component-wise non-negativity of the initial vector is preserved in time for the approximated solution. Several NSFD schemes have been constructed in the literature (see, for example, [1, 25, 29, 39-42]).

Proposition 1 (positivity). Suppose $V_{j-1}^{n}, V_{j}^{n}$, and $V_{j+1}^{n}$ are the real numbers that are nonnegative, in this case, method (18) offers a nonnegative approximation of $V_{j}^{n+1}$ for the solution of equation (2).

Proof. Since the parameter

$$
S, t, T, r, h, \Delta \tau, \sigma_{0} \geq 0,
$$

also

$$
\sigma^{2} \geq 0
$$

then

$$
x_{j}^{n}, y_{j}^{n}, z_{j}^{n} \geq 0
$$

Therefore, this completes the proof.
Lemma 2 (stability). The new NSFD method is unconditionally stable.

Proof. As in [5], for each $n=0,1, \ldots, M-1$, we know $V^{n+1}=\left(V_{0}^{n},\left(V^{n}\right)^{T}, V_{M}^{n}\right)$, that $V^{n+1}$ is the solution of the NSFD method, and then for the initial and boundary conditions, $L_{1}, L_{2}$, and $L_{3}$ are defined, and $V^{n+1}$ just shows

$$
\left\|V^{n+1}\right\|_{\infty} \leq \max \left\{\left\|L_{1}\right\|_{\infty},\left\|L_{2}\right\|_{\infty},\left\|L_{3}\right\|_{\infty}\right\}
$$

$\|.\|_{\infty}$ represents infinite norm. For each $n=0,1, \ldots, N-$ 1 and $j=1, \ldots, M-1$, we have

$$
V_{j}^{n+1}=x_{j}^{n} V_{j-1}^{n}+y_{j}^{n} V_{j}^{n}+z_{j}^{n} V_{j+1}^{n} .
$$
have

From (23)-(25), it is clear that $x_{j}^{n}, y_{j}^{n}, z_{j}^{n} \geq 0$, so we will

$$
\begin{aligned}
\left|V_{j}^{n+1}\right| & \leq x_{j}^{n}\left|V_{j-1}^{n}\right|+y_{j}^{n}\left|V_{j}^{n}\right|+z_{j}^{n}\left|V_{j+1}^{n}\right| \\
& \leq x_{j}^{n}\left\|V^{n}\right\|+y_{j}^{n}\left\|V^{n}\right\|+z_{j}^{n}\left\|V^{n}\right\| \\
& =\left(x_{j}^{n}+y_{j}^{n}+z_{j}^{n}\right)\left\|V^{n}\right\| .
\end{aligned}
$$

Now, for $j=0,1, \ldots, M$, let us consider the following two modes:

(i) Assume $j=1, \ldots, M-1$, then $\left\|V^{n+1}\right\|_{\infty}=\left|V_{k}^{n+1}\right|$ $k \in\{1,2, \ldots, M-1\}$ and since $r \geq 0$, 


$$
x_{k}^{n}+y_{k}^{n}+z_{k}^{n}=\frac{(1 / 2)\left(\sigma_{k} S_{k} / h\right)^{2}+(1 / \Delta \tau)+\left(r S_{k} / h\right)+(1 / 2)\left(\sigma_{k} S_{k} / h\right)^{2}}{(1 / \Delta \tau)+\left(r S_{k} / h\right)+\left(\sigma_{k} S_{k} / h\right)^{2}+r} \leq 1
$$

Therefore,

$$
\begin{aligned}
\left\|V^{n+1}\right\|_{\infty} & \leq\left(x_{k}^{n}+y_{k}^{n}+z_{k}^{n}\right)\left\|V^{n}\right\|_{\infty} \leq\left\|V^{n}\right\|_{\infty} \leq\left\|V^{n-1}\right\|_{\infty} \\
& \leq \cdots \leq\left\|V^{0}\right\|_{\infty} \leq\left\|L_{1}\right\|_{\infty} .
\end{aligned}
$$

(ii) Suppose that $j=0, M$, then if $\left\|V^{n+1}\right\|_{\infty}=\left|V_{0}^{n+1}\right|$ or $\left\|V^{n+1}\right\|_{\infty}=\left|V_{M}^{n+1}\right|$, it is seen from the boundary conditions that

$$
\left\|V^{n+1}\right\|_{\infty} \leq \max \left\{\left|V_{0}^{n+1}\right|,\left|V_{M}^{n+1}\right|\right\} \leq \max \left\{\left\|L_{2}\right\|_{\infty},\left\|L_{3}\right\|_{\infty}\right\}
$$

By combining two states (43) and (44), stability is achieved and this completes the proof.

Lemma 3 (consistency). The proposed method (18) is consistent, and its local truncation error is $\mathcal{O}\left(\Delta \tau, h^{2}\right)$.

Proof. The local truncation error for (18) is as follows:

$$
\begin{aligned}
T_{j}^{n}= & -\frac{V\left(S_{j}, \tau_{n+1}\right)-V\left(S_{j}, \tau_{n}\right)}{\Delta \tau}+r S_{j} \frac{V\left(S_{j+1}, \tau_{n}\right)-V\left(S_{j}, \tau_{n+1}\right)}{h} \\
& +\frac{1}{2} \sigma_{j}^{2} S_{j}^{2} \frac{V\left(S_{j-1}, \tau_{n}\right)-2 V\left(S_{j}, \tau_{n+1}\right)+V\left(S_{j+1}, \tau_{n}\right)}{h^{2}}-r V\left(S_{j}, \tau_{n+1}\right) .
\end{aligned}
$$

We have Taylor expansions

$$
\begin{aligned}
& V\left(S_{j}, \tau_{n+1}\right)=V\left(S_{j}, \tau_{n}\right)+\Delta \tau\left(\frac{\partial V}{\partial \tau}\right)_{j}^{n}+\frac{\Delta \tau^{2}}{2}\left(\frac{\partial^{2} V}{\partial \tau^{2}}\right)_{j}^{n}+\frac{\Delta \tau^{3}}{6}\left(\frac{\partial^{3} V}{\partial \tau^{3}}\right)_{j}^{n}+\cdots, \\
& V\left(S_{j+1}, \tau_{n}\right)=V\left(S_{j}, \tau_{n}\right)+h\left(\frac{\partial V}{\partial S}\right)_{j}^{n}+\frac{h^{2}}{2}\left(\frac{\partial^{2} V}{\partial S^{2}}\right)_{j}^{n}+\frac{h^{3}}{6}\left(\frac{\partial^{3} V}{\partial S^{3}}\right)_{j}^{n}+\cdots, \\
& V\left(S_{j-1}, \tau_{n}\right)=V\left(S_{j}, \tau_{n}\right)-h\left(\frac{\partial V}{\partial S}\right)_{j}^{n}+\frac{h^{2}}{2}\left(\frac{\partial^{2} V}{\partial S^{2}}\right)_{j}^{n}-\frac{h^{3}}{6}\left(\frac{\partial^{3} V}{\partial S^{3}}\right)_{j}^{n}+\cdots,
\end{aligned}
$$

replacing these expressions in the term $T_{j}^{n}$ as follows:

$$
\begin{aligned}
T_{j}^{n}= & \left(-\frac{\partial V}{\partial \tau}+r S \frac{\partial V}{\partial S}+\frac{1}{2} \sigma^{2} S^{2} \frac{\partial^{2} V}{\partial S^{2}}-r V\right)_{j}^{n} \\
& -\left(r j \Delta \tau+\sigma^{2} j^{2} \Delta \tau+r \Delta \tau\right)\left(\frac{\partial V}{\partial \tau}\right)_{j}^{n}+\frac{1}{2} r j h^{2}\left(\frac{\partial^{2} V}{\partial S^{2}}\right)_{j}^{n} \\
& -\left(\frac{1}{2} \Delta \tau+\frac{1}{2} r j \Delta \tau^{2}+\frac{1}{2} \sigma^{2} j^{2} \Delta \tau^{2}+\frac{1}{2} r \Delta \tau^{2}\right)\left(\frac{\partial^{2} V}{\partial \tau^{2}}\right)_{j}^{n}+\cdots
\end{aligned}
$$

However, $V$ is the solution of the Black-Scholes equation, so the proposed method is consistent:

$$
\left(-\frac{\partial V}{\partial \tau}+r S \frac{\partial V}{\partial S}+\frac{1}{2} \sigma^{2} S^{2} \frac{\partial^{2} V}{\partial S^{2}}-r V\right)_{j}^{n}=0
$$

Therefore, the main part of the local truncation error is as follows:

$$
-\left(r j \Delta \tau+\sigma^{2} j^{2} \Delta \tau+r \Delta \tau\right)\left(\frac{\partial V}{\partial \tau}\right)_{j}^{n}+\frac{1}{2} r j h^{2}\left(\frac{\partial^{2} V}{\partial S^{2}}\right)_{j}^{n}
$$

so $\mathcal{O}\left(\Delta \tau, h^{2}\right)$. Therefore, this completes the proof.

Theorem 1. The solution of method (18) convergences to the viscosity solution of equation (2), when

$$
\begin{aligned}
h & =\max _{0 \leq j \leq M-1} h_{j} \longrightarrow 0, \\
\Delta \tau & =\max _{0 \leq n \leq N-1} \Delta \tau_{n} \longrightarrow 0 .
\end{aligned}
$$

Proof. If the discretized method for nonlinear partial differential equations be stable, consistent, and monotone, then 

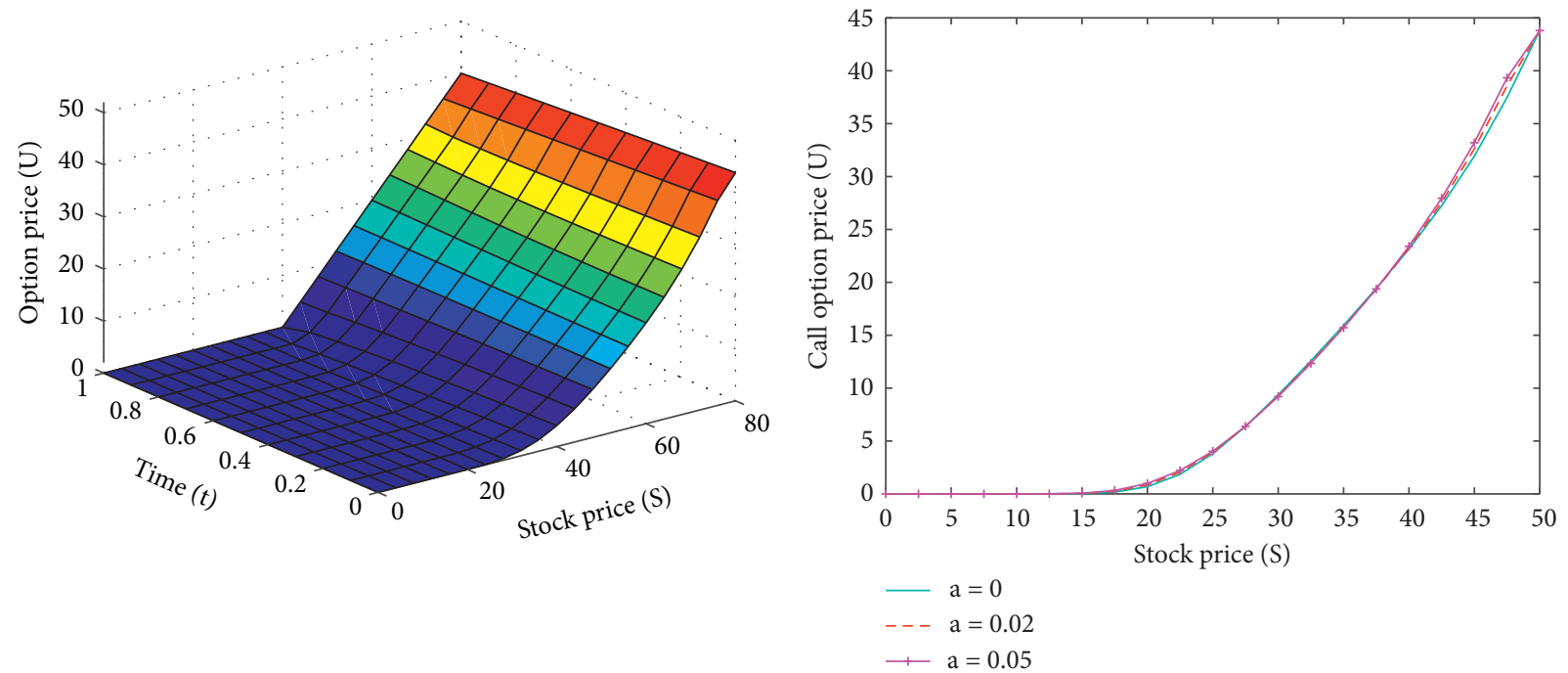

(a)

(b)

FiguRE 1: (a) European call option price charts of the explicit nonstandard method for $a=0.05$ and (b) European option price charts of the explicit nonstandard method for different transaction costs in $t=0$.

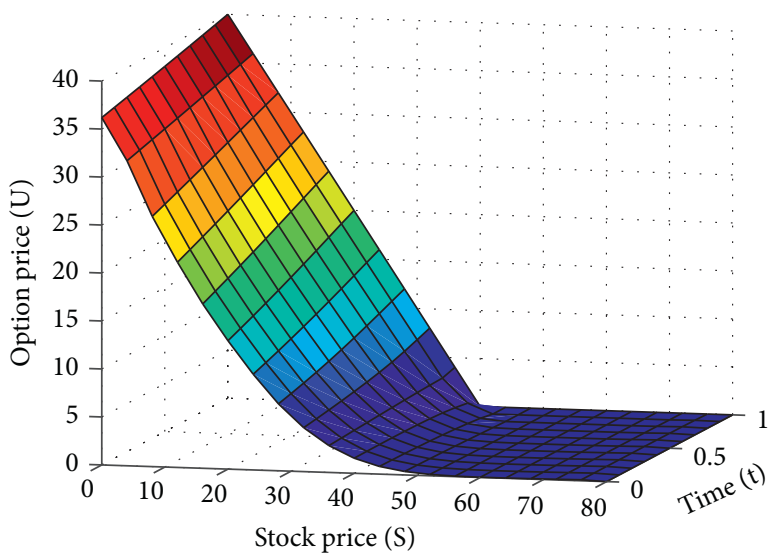

(a)

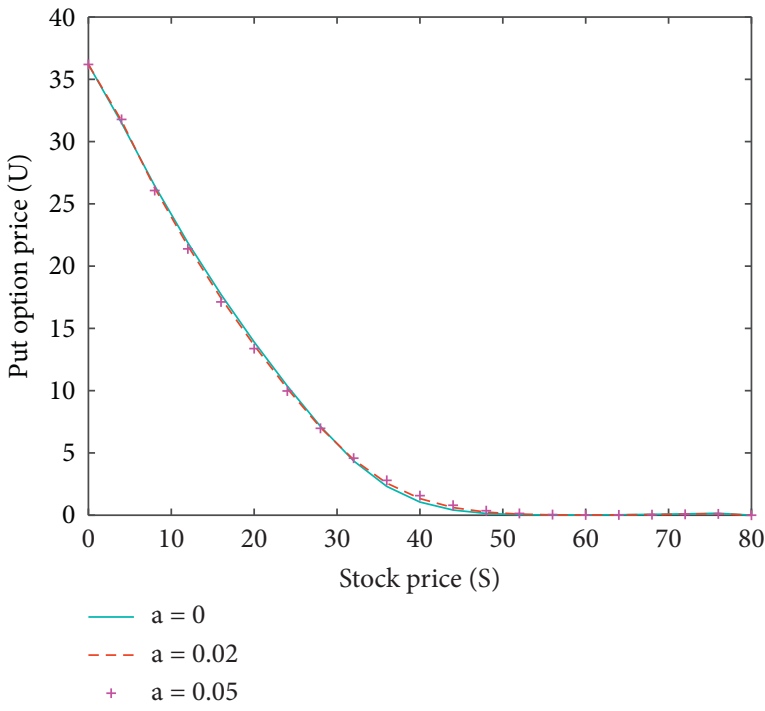

(b)

FIGURE 2: (a) European put option price charts of the explicit nonstandard method for $a=0.05$ and (b) European option price charts of the explicit nonstandard method for different transaction costs in $t=0$.

the approximate solution converges to the viscosity solution of the equation [43].

\section{Numerical Results}

To illustrate the effectiveness of the new NSFD, the problem in the independent variables $(S, \tau)$ is solved for various values of parameter transaction cost $a$ on a number of uniform meshes by the numerical method presented in the previous sections. The numerical solutions are then transformed back in $(S, t)$. We have programmed these methods in MATLAB.

Example 1. The first test problem is which we consider European call option contracts for solving equation (6) with initial (8) and boundary conditions (9) and (10) by the following parameters: 

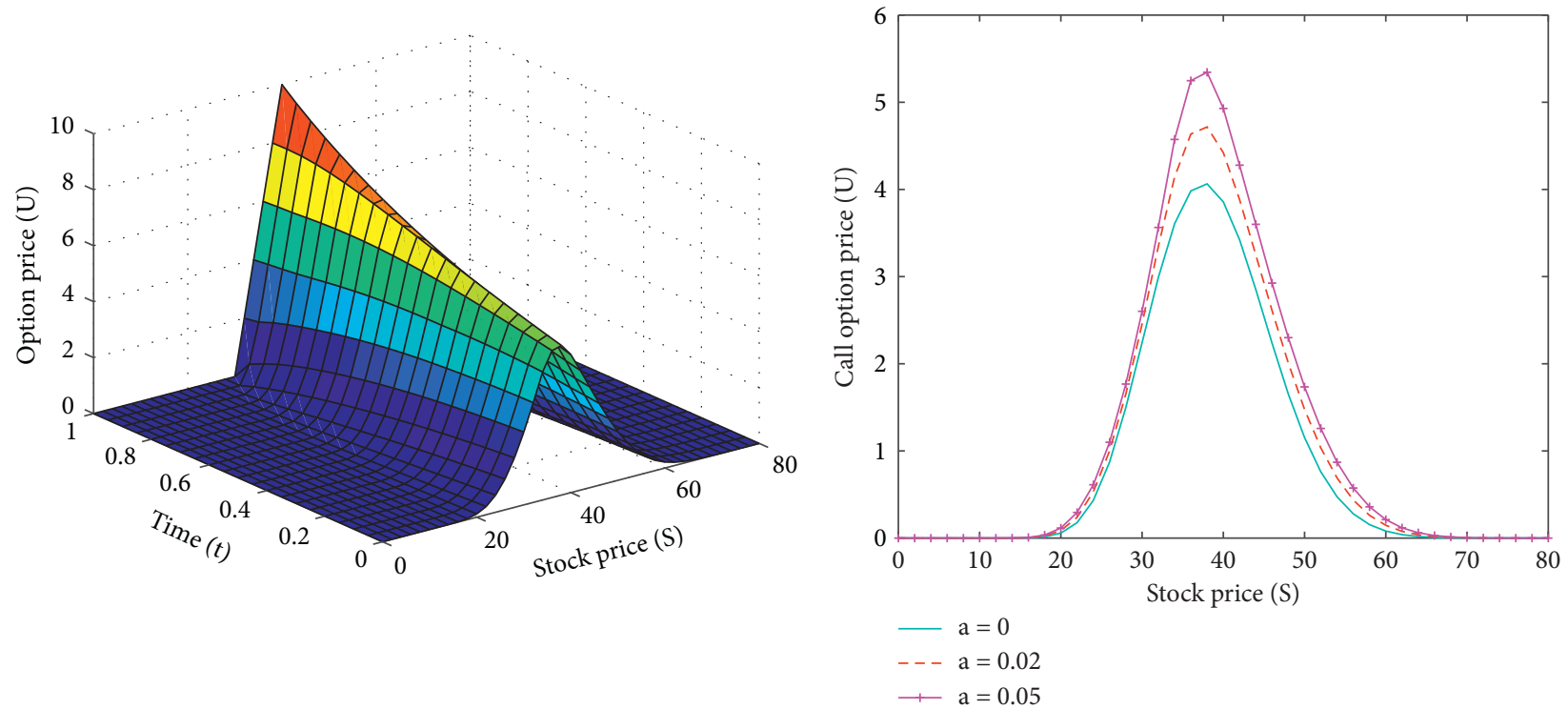

(a)

(b)

FIGURE 3: (a) Butterfly spread option price charts of the explicit nonstandard method for $a=0.05$ and (b) butterfly spread option price charts of the explicit nonstandard method for different transaction costs in $t=0$.

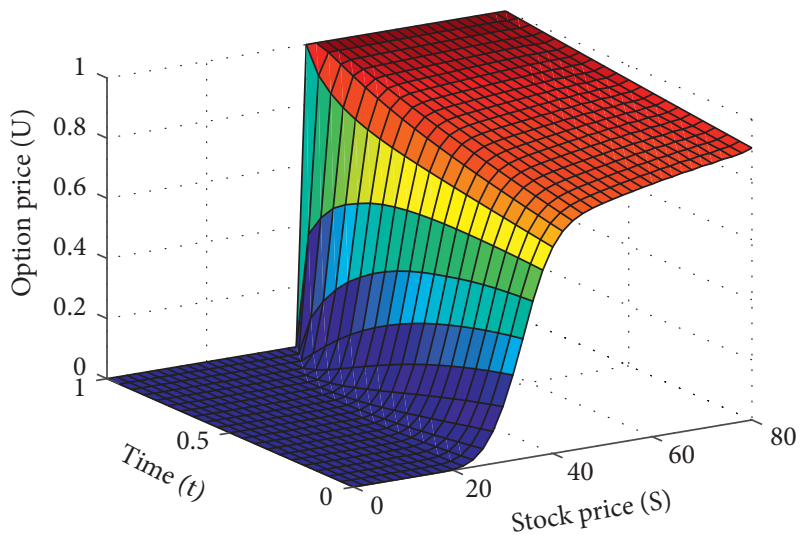

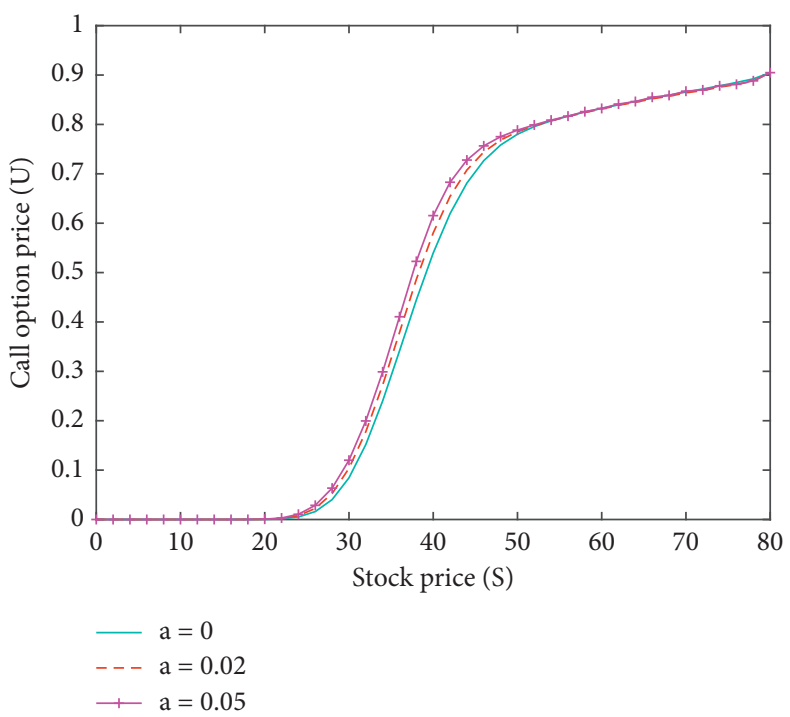

(b)

FIgURE 4: (a) European cash-or-nothing option price charts of the explicit nonstandard method for $a=0.05$ and (b) cash-or-nothing charts of the explicit nonstandard method for different transaction costs in $t=0$.

$$
\begin{aligned}
r & =0.1, \\
a & =0.05, \\
\sigma & =0.2, \\
K & =40, \\
T & =1, \\
S_{\max } & =80 .
\end{aligned}
$$

Option value with uniform meshes $h=4$ and $\Delta t=0.1$ is drawn up in Figure 1(a). It can be seen that the solution of the proposed method is positive and numerically stable. Also, the comparison of price options with different transaction costs at $t=0$ is shown in Figure 1(b). It can be seen that with the increase in the transaction cost, the price of the option will also increase. This is also true in practice. The value of the parameters used in this section has been taken from [5]. 
Example 2. As our the second numerical experiment, consider put option contracts for solving equation (6) with initial (8) and boundary conditions (9) and (10) by the following parameters:

$$
\begin{aligned}
K_{1} & =30, \\
K_{2} & =40, \\
K_{3} & =50, \\
r & =0.1, \\
\sigma_{0} & =0.2, \\
T & =1, \\
S_{\max } & =80 .
\end{aligned}
$$

Option value with uniform meshes $h=2$ and $\Delta t=0.05$ is drawn up in Figure 2(a). As can be seen from Figure 2, the function of the value of the option is an increasing function of the transaction cost parameter. Also, the price of the put option with different transaction costs at $t=0$ is shown in Figure 2(b).

Example 3. Let us consider European butterfly spread option contracts for solving equation (6) with initial (8) and boundary conditions (9) and (10) by the following parameters:

$$
\begin{aligned}
r & =0.1, \\
a & =0.05, \\
\sigma & =0.2, \\
K & =40, \\
T & =1, \\
S_{\max } & =80 .
\end{aligned}
$$

Option value with uniform meshes $h=4$ and $\Delta t=0.1$ is drawn up in Figure 3(a). As can be seen from Figure 3, the function of the value of the option is an increasing function and is the transaction cost parameter. Also, the price of the European put option with different transaction costs at $t=0$ is shown in Figure 3(b).

Example 4. In our last example, we consider cash-ornothing option contracts for solving equation (6) with initial (8) and boundary conditions (9) and (10) by the following parameters:

$$
\begin{aligned}
r & =0.1, \\
\sigma_{0} & =0.2, \\
K & =40, \\
T & =1, \\
B & =1, \\
S_{\max } & =80 .
\end{aligned}
$$

Option value with uniform meshes $h=2$ and $\Delta t=0.05$ is drawn up in Figure 4(a). As can be seen from Figure 4, the function of the value of the option is an increasing function of the transaction cost parameter. Also, the price of a cashor-nothing option with different transaction costs at $t=0$ is shown in Figure 4(b).

\section{Discussion and Conclusion}

In this paper, we proposed a new nonstandard finite difference method for the Black-Scholes nonlinear equation under transaction costs, which, in addition to being free from spurious oscillation and preserving positivity property for the desired step size, is unconditionally stable. Also, we showed the convergence of the solution of the new method to the viscosity solution of the equation by proving stability, consistency, and monotonicity and observed that the price of a European option contract is an increasing function of the parameter transaction cost. Our results indicate that a properly implemented version of our scheme is useful for the numerical integration of the considered Black-Scholes equation. In our future research, we intend to solve time fractional Black-Scholes European option pricing equation using the two-scale fractal derivatives.

\section{Data Availability}

The data used to support this study are available from the corresponding author upon request.

\section{Conflicts of Interest}

The authors declare that they have no conflicts of interest.

\section{References}

[1] R. C. Merton, "Theory of rational option pricing," The Bell Journal of Economics and Management Science, vol. 4, no. 1, pp. 141-183, 1973.

[2] T. Hoggard, A. W. Whalley, and P. Wilmott, "Hedging option portfolios in the presence of transaction costs," Adv. Futures Options Res, vol. 7, pp. 21-35, 1994.

[3] M. Jandačka, "And Ševčovič, on the risk-adjusted pricingmethodology-based valuation of vanilla options and explanation of the volatility smile," Journal of Applied Mathematics, vol. 3, pp. 235-258, 2005.

[4] H. E. Leland, "Option pricing and replication with transactions costs," The Journal of Finance, vol. 40, no. 5, pp. 1283-1301, 1985.

[5] D. C. Lesmana and S. Wang, "An upwind finite difference method for a nonlinear Black-Scholes equation governing European option valuation under transaction costs," Applied Mathematics and Computation, vol. 219, no. 16, pp. 88118828, 2013.

[6] R. Company, L. Jódar, and J.-R. Pintos, "A numerical method for European Option Pricing with transaction costs nonlinear equation," Mathematical and Computer Modelling, vol. 50, no. 5-6, pp. 910-920, 2009.

[7] S. Zhou, W. Li, Y. Wei, and C. Wen, "A positivity-preserving numerical scheme for nonlinear option pricing models," Journal of Applied Mathematics, vol. 2012, Article ID 205686, 20 pages, 2012. 
[8] S. Abbas, M. Benchohra, N. Hamidi, and J. J. Nieto, "Hilfer and Hadamard fractional differential equations in Fréchet spaces," TWMS Journal of Pure and Applied Mathematics, vol. 10, no. 1, pp. 102-116, 2019.

[9] S. Harikrishnan, K. Kanagarajan, and E. M. Elsayed, "Existence and stability results for differential equations with complex order involving Hilfer fractional derivative," TWMS Journal of Pure and Applied Mathematics, vol. 10, no. 1, pp. 94-101, 2019.

[10] J.-H. He, F.-Y. Ji, and H. Mohammad-Sedighi, "Difference equation vs differential equation on different scales," International Journal of Numerical Methods for Heat \& Fluid Flow, vol. 31, no. 1, pp. 391-401, 2020.

[11] N. Moshtaghi and A. Saadatmandi, "Numerical solution for diffusion equations with distributed-order in time based on sinc-legendre collocation method," Applied and Computational Mathematics, vol. 19, no. 3, pp. 317-355, 2020.

[12] V. Gülkaç, "The homotopy perturbation method for the Black Scholes equation," Journal of Statistical Computation and Simulation, vol. 80, no. 12, pp. 1349-1354, 2010.

[13] J.-H. He, "Homotopy perturbation method: a new nonlinear analytical technique," Applied Mathematics and Computation, vol. 135, no. 1, pp. 73-79, 2003.

[14] K. Trachoo, W. Sawangtong, and P. Sawangtong, "Laplace transform homotopy perturbation method for the two dimensional Black Scholes model with European call Option," Mathematical and Computational Applications, vol. 22, pp. 1-11, 2017.

[15] J.-H. He and X.-H. Wu, "Variational iteration method: new development and applications," Computers \& Mathematics with Applications, vol. 54, no. 7-8, pp. 881-894, 2007.

[16] P. Kashyap and A. Kumar Sharma, "Numerical solution of Black-Scholes equation using variational iteration method," International Journal of Innovative Research in Science, Engineering and Technology, vol. 9, no. 1, pp. 12-19, 2020.

[17] H. Rouhparvary and M. Salamatbakhshz, "Analytical solution of the Black-Scholes equation by using variational iteration method," Applied Mathematics E-Notes, vol. 13, pp. 243-248, 2013.

[18] S. Mashayekhi and J. hugger, "Finite difference schemes for a nonlinear black-scholes model with transaction cost and volatility risk," Acta Mathematica Universitatis Comenianae, vol. 2, pp. 255-266, 2015.

[19] B. Düring, M. Fournié, and A. Jüngel, "High order compact finite difference schemes for a nonlinear Black-Scholes equation," International Journal of Theoretical and Applied Finance, vol. 6, no. 7, pp. 767-789, 2003.

[20] P. Heider, "Numerical methods for non-linear black-scholes equations," Applied Mathematical Finance, vol. 17, no. 1, pp. 59-81, 2010.

[21] M. N. Koleva and L. G. Vulkov, "Fast computational approach to the Delta Greek of non-linear Black-Scholes equations," Journal of Computational and Applied Mathematics, vol. 340, pp. 508-522, 2018.

[22] S. Gulen, C. Popescu, and M. Sari, "A new approach for the black-scholes model with linear and nonlinear volatilities," Mathematics, vol. 7, no. 8, p. 760, 2019.

[23] A. Ghanadian and T. Lotfi, "Approximate solution of nonlinear Black-Scholes equation via a fully discretized fourthorder method," AIMS Mathematics, vol. 5, no. 2, pp. 879-893, 2020.

[24] M. R. Grossinho, Y. F. Kord, and D. Sevcovic, "Pricing American call options using the Black-Scholes equation with a nonlinear volatility function," Journal of Computational Finance, vol. 23, no. 4, pp. 93-113, 2020.

[25] R. E. Mickens, Nonstandard Finite Difference Models of Differential Equations, World Scientific, Singapore, 1994.

[26] M. Mehdizadeh Khalsaraei and R. Shokri Jahandizi, "A modified explicit method for the Black-Scholes equation with positivity preserving property," Journal of Mathematical and Computational Science, vol. 15, pp. 299-305, 2015.

[27] M. Mehdizadeh Khalsaraei and R. Shokri Jahandizi, "A positivity preserving schemes for black-scholes equation," Journal of Finance and Accounting, vol. 6, pp. 101-105, 2015.

[28] M. Mehdizadeh Khalsaraei and R. Shokri Jahandizi, "An efficient nonstandard numerical method with positivity preserving property," Journal of Mathematical Modeling, vol. 4, no. 2, pp. 161-169, 2016.

[29] M. M. Khalsaraei, A. Shokri, H. Ramos, and S. Heydari, "A positive and elementary stable nonstandard explicit scheme for a mathematical model of the influenza disease," Mathematics and Computers in Simulation, vol. 182, pp. 397-410, 2021.

[30] M. M. Khalsaraei, "Positivity of an explicit Runge-Kutta method," Ain Shams Engineering Journal, vol. 6, no. 4, pp. 1217-1223, 2015.

[31] M. M. Khalsaraei and R. S. Jahandizi, “A family of positivity preserving schemes for numerical solution of Black-Scholes equation," International Journal of Financial Engineering, vol. 3, no. 4, p. 1650025, 2016.

[32] M. Mehdizadeh Khalsaraei and R. Shokri Jahandizi, "Efficient explicit nonstandard finite difference scheme with positivitypreserving property," Journal of Science, vol. 30, no. 1, pp. 259-268, 2017.

[33] R. Company, E. Navarro, J. Ramón Pintos, and E. Ponsoda, "Numerical solution of linear and nonlinear Black-Scholes option pricing equations," Computers \& Mathematics with Applications, vol. 56, no. 3, pp. 813-821, 2008.

[34] M. Mehdizadeh Khalsaraei, "An improvement on the positivity results for 2-stage explicit Runge-Kutta methods," Journal of Computational and Applied Mathematics, vol. 235, no. 1, pp. 137-143, 2010.

[35] M. Mehdizadeh Khalsaraei and N. Usmani, "A family of positive nonstandard numerical methods with application to Black-Scholes equation," Sahand Communications in Mathematical Analysis, vol. 5, no. 1, pp. 31-40, 2017.

[36] M. Milev and A. Tagliani, "Low volatility options and numerical diffusion of finite difference schemes," Serdica Mathematical Journal, vol. 36, no. n.3, pp. 223-236, 2010.

[37] M. Milev and A. Tagliani, "Nonstandard finite difference schemes with application to finance: option pricing," Serdica Mathematical Journal, vol. 36, no. 1, pp. 75-88, 2010.

[38] M. Milev and A. Tagliani, "Numerical valuation of discrete double barrier options," Journal of Computational and Applied Mathematics, vol. 233, no. 10, pp. 2468-2480, 2010.

[39] M. M. Khalsaraei and F. Khodadosti, "Qualitatively stability of nonstandard 2-stage explicit Runge-Kutta methods of order two," Computational Mathematics and Mathematical Physics, vol. 56, no. 2, pp. 235-242, 2016.

[40] R. E. Mickens and P. M. Jordan, "A new positivity-preserving nonstandard finite difference scheme for the DWE," $\mathrm{Nu}$ merical Methods for Partial Differential Equations, vol. 21, no. 5, pp. 976-985, 2005.

[41] R. E. Mickens and P. M. Jordan, "A positivity-preserving nonstandard finite difference scheme for the Damped Wave Equation," Numerical Methods for Partial Differential Equations, vol. 20, no. 5, pp. 639-649, 2004. 
[42] M. Milev and A. Tagliani, "Efficient implicit scheme with positivity preserving and smoothing properties," Journal of Computational and Applied Mathematics, vol. 243, pp. 1-9, 2013.

[43] G. Barles, "Convergence of numerical schemes for degenerate parabolic equations arising in finance," in Numerical Methods in Finance, L. C. G. Rogers and D. Talay, Eds., Cambridge University Press, Cambridge, UK, 1997. 